\title{
Hardness of metallic crystals
}

\author{
WUHUI LI ${ }^{\dagger}$, FENGZHANG REN ${ }^{\dagger \dagger}, *$, JUANHUA SU, ZHANHONG MA, \\ KE $\mathrm{CAO}^{\dagger}$ and BAOHONG TIAN \\ School of Materials Science and Engineering, Henan University of Science and Technology, \\ Luoyang 471003, China \\ ${ }^{\dagger}$ Shenyang National Laboratory for Materials Science, Institute of Metal Research, Chinese Academy of Sciences, \\ Shenyang 110016, China \\ ${ }^{\dagger \dagger}$ Henan Key Laboratory of Advanced Non-ferrous Metals, Luoyang 471003, China
}

MS received 26 February 2010; revised 22 March 2010

\begin{abstract}
This paper presents a new formula for calculating the hardness of metallic crystals, resulted from the research on the critical grain size with stable dislocations. The formula is $H=6 k G /\left[\pi(1-v) e^{\eta}\right]$, where $H$ is the hardness, $k$ the coefficient, $G$ the shear modulus, $v$ the Poisson's ratio, $\eta$ a function of the radius of an atom $(r)$ and the electron density at the atom interface $(n)$. The formula will not only be used to testify the critical grain size with stable dislocations, but also play an important role in the understanding of mechanical properties of nanocrystalline metals.
\end{abstract}

Keywords. Hardness; metallic crystals; dislocation; electron density.

\section{Introduction}

The well-known Hall-Petch relationship predicts that the strength or hardness of conventional metal alloys increases with decreasing grain sizes. However, the relationship fails when the grain size is down to nanometers as many experimental results have shown (EI-Sherik et al 1992; Ren et al 2004; Siegel and Fougere 1995; EISherik et al 2005). In order to explain this phenomenon, the critical grain sizes with stable dislocations (the critical sizes of dislocation hereafter) in some metals were brought forward. Gryaznov et al (1989) raised the critical sizes of dislocation in 5 kinds of metals (Gryaznov et al 1989) and modified the critical sizes of dislocation (Gryaznov et al 1991). They found that the characteristic length of dislocation stability in $\mathrm{Cu}$ and $\mathrm{Ni}$ are $38 \mathrm{~nm}$ (sphere) or $24 \mathrm{~nm}$ (cylinder) and $16 \mathrm{~nm}$ (sphere) or $10 \mathrm{~nm}$ (cylinder), respectively. Nieh and Wadsworth (1991) provided the critical sizes of dislocation in 6 kinds of metals and compounds; the sizes of $\mathrm{Cu}$ and $\mathrm{Ni}$ are $19.3 \mathrm{~nm}$ and $2.5 \mathrm{~nm}$, respectively. Wang et al (1995) defined the critical sizes of dislocation in 4 kinds of metals; the size of $\mathrm{Cu}$ and $\mathrm{Ni}$ are 1.7-39.4 $\mathrm{nm}$ and 1.3-25.6 nm, respectively. Cheng and Cheng (2001) declared the critical size of dislocation in 15 kinds of metals, the size of $\mathrm{Cu}$ and $\mathrm{Ni}$ are $1.84 \mu \mathrm{m}$ and $3.18 \mu \mathrm{m}$, respectively. The different author's critical sizes of dislocation are listed in table 1 . As

\footnotetext{
*Author for correspondence (lyrenfz@163.com)
}

shown in table 1, Cheng's results are far away from others'.

The physical measurements of the critical sizes of dislocation of these metals were provided by Misra et al (1998), Ke et al (1995) and Schuh et al (2002) in their articles (Misra et al 1998; Ke et al 1995; Schuh et al 2002). The values did not agree with these abovecalculated values and the accuracy and uncertainties of the measured values are not known either. However, the measured values are in the same range of the $10-50 \mathrm{~nm}$, more or less the same as most authors, but far away from Cheng's results. Since Cheng's method seems reasonable, efforts were made to check how to modify Cheng's formulas and parameters and thus the results would be similar to the measured values. This is a big challenge. The four authors (table 1) all got the critical sizes of dislocation for some metals; Cheng's deduction and Nieh's deduction were modified to derive a new formula of the hardness of metallic crystals for the above purpose.

\section{Formulae}

\subsection{Cheng's derivation process of the critical grain size}

Cheng and Cheng (2001) thought that an elastic stress field will exist around dislocations after they are formed. The attraction pressure $P$ produced by the potential of the inner core balances the repulsive pressure. On removing the ion core, electrons will be induced to the external boundary of the void, thus producing an attraction 
Table 1. The critical sizes of dislocation from different authors.

\begin{tabular}{|c|c|c|c|c|c|}
\hline \multirow[b]{2}{*}{ Material } & \multicolumn{4}{|c|}{ Calculated (nm) } & \multirow[b]{2}{*}{ Measured (nm) } \\
\hline & Gryaznov & Nieh & Wang & Cheng & \\
\hline $\mathrm{Al}$ & 11,18 & - & $1 \cdot 2-59 \cdot 3$ & 6960 & - \\
\hline $\mathrm{Cu}$ & 24,38 & $19 \cdot 3$ & $1 \cdot 7-39 \cdot 4$ & 1840 & < 50 (Misra et al 1998) \\
\hline $\mathrm{Ni}$ & 10,16 & $2 \cdot 5$ & $1 \cdot 3-25 \cdot 6$ & 3180 & $12-14$ (Schuh et al 2002) \\
\hline $\mathrm{Pd}$ & - & $11 \cdot 2$ & $1 \cdot 8-75 \cdot 3$ & - & - \\
\hline$\alpha-\mathrm{Fe}$ & 2,3 & - & - & 1000 & - \\
\hline $\mathrm{Au}$ & - & - & - & 250 & $10($ Ke et al 1995) \\
\hline
\end{tabular}

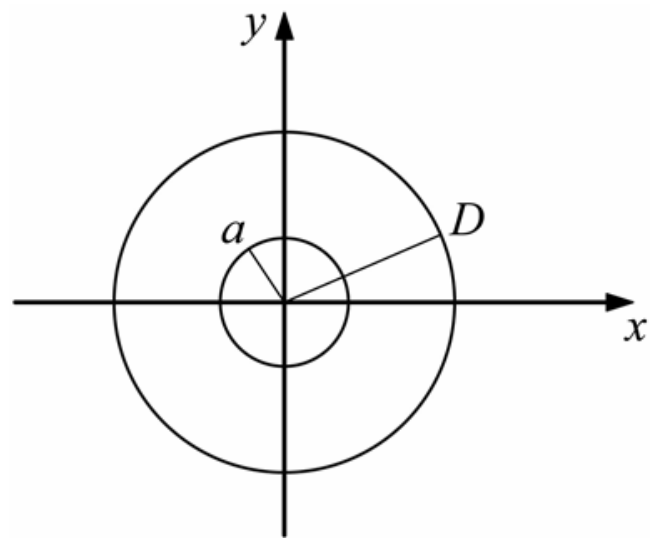

Figure 1. Schematic diagram of an edge dislocation in the $x-y$ plane (Cheng and Cheng 2001).

counterbalancing the expanding pressure of the shell of electrons. This shows that only part of the total volume of the void is occupied by electrons. The size of an edge dislocation can be described by the outer and inner radii $D$ and $a$, respectively, as shown in figure 1 .

The elastic energy per unit length $E_{\mathrm{L}}$ of the dislocation between $D$ and $a$ is given as

$$
E_{\mathrm{L}}=\frac{\mu b^{2}}{4 \pi(1-v)}\left[\ln \frac{D}{a}-\frac{D^{2}-a^{2}}{D^{2}+a^{2}}\right], \quad D \gg a,
$$

where $\mu$ is the shear modulus, $b$ the Burgers vector and $v$ the Poisson's ratio.

According to solid physics, the pressure of electrons gas $P$ is given by

$$
3 P=2 K+V_{\mathrm{ex}},
$$

where $K$ is the kinetic energy density of electrons at the boundary of atoms, and $V_{\text {ex }}$ the exchange energy density. The pressure $P$ can be explicitly written as

$$
P=\frac{1}{3}\left\{\frac{2 \times 3 h^{2}}{10 m}\left(\frac{3}{8 \pi}\right)^{2 / 3} n^{5 / 3}-\frac{1}{4 \pi \varepsilon_{0}} e^{2}\left(\frac{3}{\pi}\right)^{1 / 3} \frac{3}{4} n^{4 / 3}\right\},
$$

where $n$ is the electron density at interfaces of atoms, $m$ the mass of electron, $h$ the Plank constant, $\varepsilon_{0}$ the permittivity of vacuum and $e$ the electron charge.
Work $E_{\mathrm{P}}$ is done by this pressure in the creation of holes of dislocation per unit length with a volume $V$ in every plane

$$
E_{\mathrm{P}}=-P V / d \text {. }
$$

Here $V$ is the volume of electrons corresponding to those driven into the void of an atom of dislocation by the environment and $d$ is the distance between two neighbouring holes.

A close-packed structure with the length of each layer $d$ is considered, then

$$
d=2 r(2 / 3)^{1 / 2} \text {. }
$$

The value $V \cdot n$ is equal to the valence $z$, i.e.

$$
V \cdot n=z \text {. }
$$

The total energy $\varepsilon$ expanded in the creation of a unit length of dislocations is the sum of the values from (1) and (4)

$$
\varepsilon=E_{\mathrm{L}}+E_{\mathrm{P}}
$$

On account of the meta-stability of dislocations, $\varepsilon$ must be positive. Equation (7) is thus capable of yielding the ratio $D / a$, giving the limit in the sizes of the dislocations. Combining (1)-(7), one obtains the size limit as follows (Cheng and Cheng 2001)

$$
\begin{aligned}
& D=a e^{\eta+1}, \\
& \eta=\frac{100(1-v)}{\mu^{*}} z r^{*^{-3}} n^{*^{2 / 3}}\left(1-0 \cdot 546 n^{*-1 / 3}\right),
\end{aligned}
$$

where

$$
n^{*} \equiv n 10^{-29}, \quad r^{*} \equiv n 10^{10}, \quad b=2 r, \quad \mu^{*} \equiv \mu 10^{-10}, \quad a \cong r .
$$

In (1), it is more reasonable to substitute the total energy of an edge dislocation per unit length for the elastic energy of an edge dislocation per unit length. The total energy of an edge dislocation per unit length can be expressed as (10) (Yu 2000)

$$
E_{\mathrm{L}}=\frac{\mu b^{2}}{4 \pi(1-v)} \ln \frac{2 D}{a} .
$$

Thus we obtain (11) and (12) by combining (2)-(7) and (10) 


$$
\begin{aligned}
& D=\frac{1}{2} a e^{\eta} \\
& \eta=\frac{96 \cdot 9(1-v)}{\mu^{*}} z r^{*^{-3}} n^{*^{2 / 3}}\left(1-0 \cdot 523 n^{*^{-1 / 3}}\right) .
\end{aligned}
$$

Applying (11) and (12) to copper, assuming $z=1$, $n^{*}=2.934 \mathrm{~m}^{-3}, r^{*}=1.413 \mathrm{~m}, \mu^{*}=4.79 \mathrm{~Pa}$ (Cheng and Cheng 1996), $v=0 \cdot 31$, then $2 D=88 \mathrm{~nm}$. The calculated value is relatively close to the measured values. To nickel, assuming $z=2, n^{*}=3.195 \mathrm{~m}^{-3}, r^{*}=1.382 \mathrm{~m}, \mu^{*}=8.00 \mathrm{~Pa}$, $v=0.34$ (Cheng and Cheng 1996), then $2 D=663 \mathrm{~nm}$. This result is rather far away from the measured values. The possible reason related to the value of $n^{*}$ will be studied in more detail in our future work.

\subsection{Nieh's derivation process of the critical grain size}

There are only two edge dislocations as shown in figure 2, where $l$ is the distance between the two dislocations, $\sigma_{\text {app }}$ the externally applied stress.

The repulsive force per unit length between two edge dislocations $f$ is given by

$$
f=\frac{G b^{2}}{2 \pi(1-v)} \cdot \frac{1}{l},
$$

where $G$ is the shear modulus, $b$ the Burgers vector and $v$ Poisson's ratio. These two dislocations will move to their equilibrium positions when the repulsive force between them is cancelled out by the externally applied force $\tau_{\text {app }} b$, where $\tau_{\text {app }}$ is the applied shear stress, i.e.

$$
f=\tau_{\text {app }} b .
$$

Assuming that the hardness, $H$, is about $3 \sigma_{\text {app }}$ and $\tau_{\text {app }} \sim\left(\sigma_{\text {app }} / 2\right)$, then the equilibrium distance (also called the critical grain size), $l_{c}$, between the two edge dislocations can be obtained from the following formula (Nieh and Wadsworth 1991)

$$
l_{\mathrm{c}}=\frac{3 G b}{\pi(1-v) \cdot H} \text {. }
$$

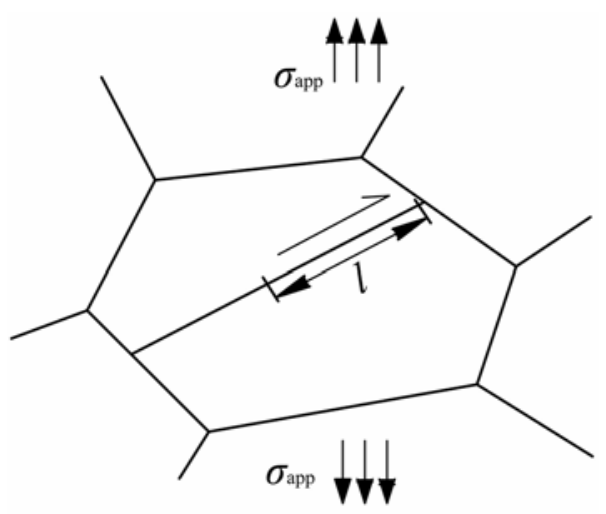

Figure 2. Dislocation pile-up at grain boundaries (Nieh and Wadsworth 1991).

\section{Results and discussion}

\subsection{The hardness formula of metallic crystals}

Equation (15) defined the critical sizes of dislocation, $l_{c}$, which is the same as $2 D$, expressed in (11). The properties, one from the force perspective, the other from energy, should be the same. Thus, a coefficient $k$ can be introduced. If $2 D=k \cdot l_{\mathrm{c}}$, then combining (11), (15) and $b=2 r$, one has

$$
H=\frac{6 k G}{\pi(1-v) e^{\eta}},
$$

where

$$
G=\mu=\frac{E}{2(1+v)},
$$

$k$ is the coefficient, $v$ the Poisson's ratio and

$$
\eta=\frac{96 \cdot 9(1-v)}{\mu^{*}} z r^{*^{-3}} n^{*^{2 / 3}}\left(1-0 \cdot 523 n^{*-1 / 3}\right) .
$$

3.2 The physical significance of the components of the hardness formula

From (16), we could know that hardness $H$ is the function of electron density $n$ and the radius of atom $r$. The relationship of $r$ and $n$ from Thomas-Fermi-Dirac (TFD) model is

$$
n(x)=\frac{z}{4 \pi \mu^{3}}\left[\varepsilon+\left(\frac{\psi}{x}\right)^{1 / 2}\right]^{3},
$$

where $\mu=a_{0}\left(9 \pi^{2} / 128 Z\right)^{1 / 3}, a_{0}$ is Bohr radius, $Z$ the atomic number, $\varepsilon=\left(3 / 32 \pi^{2}\right)^{1 / 3} Z^{-2 / 3}, x$ the dimensionless atomic radius, $r=\mu x$ the actual atomic radius, and $n(x)$ the electron density. $\psi$ is called the TFD function satisfying famous TFD equation, namely,

$$
\frac{\mathrm{d}^{2} \psi}{\mathrm{d} x^{2}}=x\left[\varepsilon+\left(\frac{\psi}{x}\right)^{1 / 2}\right]^{3} .
$$

The boundary condition of (18) is

$$
\begin{aligned}
& \psi(0)=1, \\
& x_{0} \frac{\mathrm{d} \psi\left(x_{0}\right)}{\mathrm{d} x_{0}}=\psi\left(x_{0}\right) .
\end{aligned}
$$

The relationship of $n$ and $r$ is shown in figure 3. For one certain atomic radius $r_{0}$, there exists one electron density distribution curve, $n^{\prime}-r^{\prime}$, corresponding to (17) and (18). When the atomic radius changes from $r_{0}$ to $r_{0}+\Delta r$, the relevant electron density distribution curve turns into a 


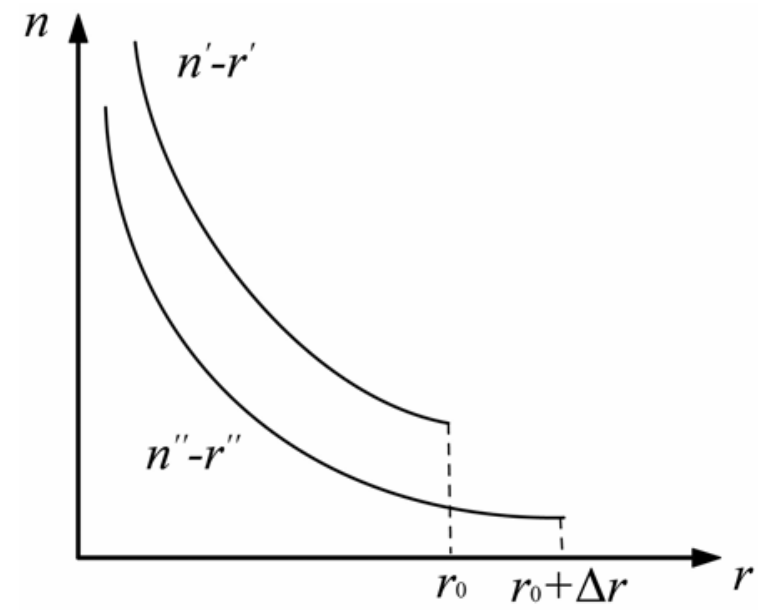

Figure 3. Effect of atomic radius variation on electron density (Li 2004)

completely new one, $n^{\prime \prime}-r^{\prime \prime}$. The relationship of $H$ and $n$ could be properly understood only after the meaning of $n$ is understood profoundly.

\subsection{The applicable range of the hardness formula}

Deducted for metallic crystals, the formula of hardness could be applied to metallic crystals only. Since $H$ and $n$ are all measurable, the coefficient $k$ could be identified. Different materials have different $k$ values, which can be calculated with sufficient experimental data. Because the nanocrystal contains no dislocation, its expending energy is zero. $H$ is the function of $n$ and $r$. When the size of the grain is less than the critical size of the dislocation, this formula could still be applied. Thus this formula is the supplement of the Hall-Petch relationship and very useful in guiding the understanding of the mechanical behaviour of the nanomaterials.

\section{Conclusions}

A new formula of the hardness of metallic crystals has been given, i.e. $H=6 k G /\left[\pi(1-v) e^{\eta}\right]$, on the deduction of
Cheng's and Nieh's. This formula will not only be used to testify the critical grain size with stable dislocations, but also play an important role in understanding the mechanical properties of nanocrystalline metals.

\section{Acknowledgements}

Financial support from the National Natural Science Foundation of China (No. 50771042), the program for Basic and Frontier Technologies Research in Henan Province (092300410064), Technological Renovation Talents in University of Henan Province (2009HASTIT023) and the program for Young Key Teacher in Henan Province (Grant No. 2005-461) are gracefully acknowledged.

\section{References}

Cheng K J and Cheng S Y 1996 Prog. Nat. Sci. 612

Cheng K J and Cheng S Y 2001 Prog. Nat. Sci. 11701

El-Sherik A M, Erb U, Palumbo G and Aust K T 1992 Scripta Metall. et Mater. 271185

El-Sherik A M, Shirokoff J and Erb U 2005 J. Alloys Compd. 389140

Gryaznov V G, Kaprelov A M and Romanov A E 1989 Scripta Metall. 231443

Gryaznov V G, Polonsky I A, Romanov A E and Trusov L I 1991 Phys. Rev. B44 42

Ke M, Hackney S A, Milligan W W and Aifantis E C 1995 Nanostruct. Mater. 5689

Li S C 2004 Prog. Nat. Sci. 14113

Misra A, Verdier M, Lu Y C, Kung H, Mitchell T E, Nastasi M and Embury J D 1998 Scripta Mater. 39555

Nieh T G and Wadsworth J 1991 Scripta Metall. et Mater. 25 955

Ren F Z, Zhou G S, Zheng M S, Zhao W Z and Gu H C 2004 Rare Metal Mat. Eng. 3933 (in Chinese)

Siegel R W and Fougere G E 1995 Nanostruct. Mater. 6205

Schuh C A, Nieh T G and Yamasaki T 2002 Scripta Mater. 46 735

Wang N, Wang Z R, Aust K T and Erb U 1995 Acta Metall. Mater. 43519

Yu Y N 2000 Metallography principles (Beijing: Metallurgical Industry Press) Ch. 6 (in Chinese) 